\title{
Germline PTEN mutations in Cowden syndrome-like families
}

\begin{abstract}
Debbie J Marsh, Patricia L M Dahia, Stacey Caron, Jennifer B Kum, Ian M Frayling, Ian P M Tomlinson, Kevin S Hughes, Rosalind A Eeles, Shirley V Hodgson, Vicky A Murday, Richard Houlston, Charis Eng
\end{abstract}

\author{
Department of Adult \\ Oncology and Charles \\ A Dana Human \\ Cancer Genetics Unit, \\ Dana-Farber Cancer \\ Institute, Department \\ of Medicine, Harvard \\ Medical School, Susan \\ and Richard Smith \\ Laboratories, SM822, \\ 1 Jimmy Fund Way, \\ Boston, MA \\ 02115-6084, USA \\ D J Marsh \\ P L M Dahia \\ $S$ Caron \\ J B Kum \\ C Eng
}

ICRF Colorectal Cancer Unit, St Mark's Hospital, Northwick Park, Middlesex, UK

I M Frayling

Tumour Genetics Group, Wellcome Trust Centre for Human Genetics, University of Oxford, UK

I P M Tomlinson

\section{Department of}

Surgery, Lahey Clinic, Burlington, MA 02115, USA

K S Hughes

\section{Section of Molecular} Carcinogenesis, Institute of Cancer Research and Cancer Genetics Clinic, Royal Marsden Hospital, Sutton, Surrey, UK R A Eeles

R Houlston

Department of Medical Genetics, United Medical and Dental Schools of Guy's and St Thomas's Hospital, London, UK $S$ V Hodgson

Department of Clinical Genetics, St George's Hospital Medical School, London, UK V A Murday

\section{Abstract}

Cowden syndrome (CS) or multiple hamartoma syndrome (MIM 158350) is an autosomal dominant disorder with an increased risk for breast and thyroid carcinoma. The diagnosis of CS, as operationally defined by the International Cowden Consortium, is made when a patient, or family, has a combination of pathognomonic major and/or minor criteria. The CS gene has recently been identified as PTEN, which maps at 10q23.3 and encodes a dual specificity phosphatase. PTEN appears to function as a tumour suppressor in CS, with between $13-80 \%$ of CS families harbouring germline nonsense, missense, and frameshift mutations predicted to disrupt normal PTEN function. To date, only a small number of tumour suppressor genes, including BRCA1, BRCA2, and p53, have been associated with familial breast or breast/ovarian cancer families. Given the involvement of PTEN in CS, we postulated that PTEN was a likely candidate to play a role in families with a "CS-like" phenotype, but not classical CS. To answer these questions, we gathered a series of patients from families who had features reminiscent of CS but did not meet the Consortium Criteria. Using a combination of denaturing gradient gel electrophoresis (DGGE), temporal temperature gel electrophoresis (TTGE), and sequence analysis, we screened 64 unrelated CS-like subjects for germline mutations in PTEN. A single male with follicular thyroid carcinoma from one of these $64(2 \%)$ CS-like families harboured a germline point mutation, c.209T $\rightarrow$ C. This mutation occurred at the last nucleotide of exon 3 and within a region homologous to the cytoskeletal proteins tensin and auxilin. We conclude that germline PTEN mutations play a relatively minor role in CS-like families. In addition, our data would suggest that, for the most part, the strict International Cowden Consortium operational diagnostic criteria for $\mathrm{CS}$ are quite robust and should remain in place. $(\Im$ Med Genet 1998;35:881-885)

Keywords: PTEN; Cowden syndrome; breast; thyroid
Breast and thyroid carcinoma are two frequently occurring neoplasms in the female population. Increased risks for both breast and thyroid cancer are prominent features of Cowden syndrome (CS). The hallmark phenotype of this inherited cancer syndrome is the presence of hamartomas, developmentally incorrect, benign, hyperplastic growths, in multiple organ systems including the skin, gastrointestinal tract, central nervous system, breast, and thyroid. Breast cancer will develop in $25-50 \%$ of women with CS and $3-10 \%$ of all CS patients will develop thyroid cancer. ${ }^{12}$ At present, only four tumour suppressor genes have been associated with familial breast cancer, BRCA1, BRCA2, p53, and PTEN. ${ }^{3-7}$ Initially thought to account for over $80 \%$ of hereditary breast cancer, ${ }^{89}$ germline mutations in BRCA1 and BRCA2 together are now thought to account for $25-50 \%$ of all familial breast cancer, ${ }^{10}$ thus opening up the possibility of other BRCAX genes. Along these lines, germline mutations in p53 are associated with $70 \%$ of cases of Li-Fraumeni syndrome, an autosomal dominant condition comprising breast cancer, brain tumours, sarcomas, and adrenocortical carcinomas. ${ }^{3411} \mathrm{Re}-$ cently, the CS susceptibility gene has been identified as the tumour suppressor gene PTEN, also known as MMAC1 and TEP1. ${ }^{72-14}$ PTEN maps to 10q23.3 and encodes a 403 amino acid dual specificity phosphatase. ${ }^{12-15}$ Germline missense and truncating mutations have been reported in between $13-80 \%$ of patients with CS. ${ }^{76-18}$ It should be noted that while initial linkage studies of 12 families with CS was highly suggestive of a single locus for $C S,{ }^{19}$ a subsequent study proposes that genetic heterogeneity may exist in $\mathrm{CS}^{16}$

At the somatic level, PTEN has been shown to be mutated or deleted in a number of human malignancies, including sporadic breast, brain, prostate, and kidney cancer cell lines, as well as in a number of primary tumours including endometrial carcinomas, glioblastomas, malignant melanoma, and thyroid and breast tumours. ${ }^{20-33}$

Given the role of PTEN in CS and the relatively large percentage of familial cases of breast cancer that are not caused by germline mutation of BRCA1, BRCA2, or p53, we sought to determine whether PTEN may be mutated in 
Table 1 Phenotypic classification of CS-like families

\begin{tabular}{ll}
\hline Phenotype of families & No of families \\
\hline Breast and thyroid carcinoma occurring together in at least one person & 22 \\
Breast and thyroid carcinoma occurring in different subjects & 32 \\
Breast carcinoma and thyroid disease (eg goitre) & 3 \\
Breast carcinoma/CS-like (eg trichilemmoma), no thyroid involvement & 6 \\
Thyroid carcinoma/CS-like, no breast involvement & 1 \\
Total & 64 \\
\hline
\end{tabular}

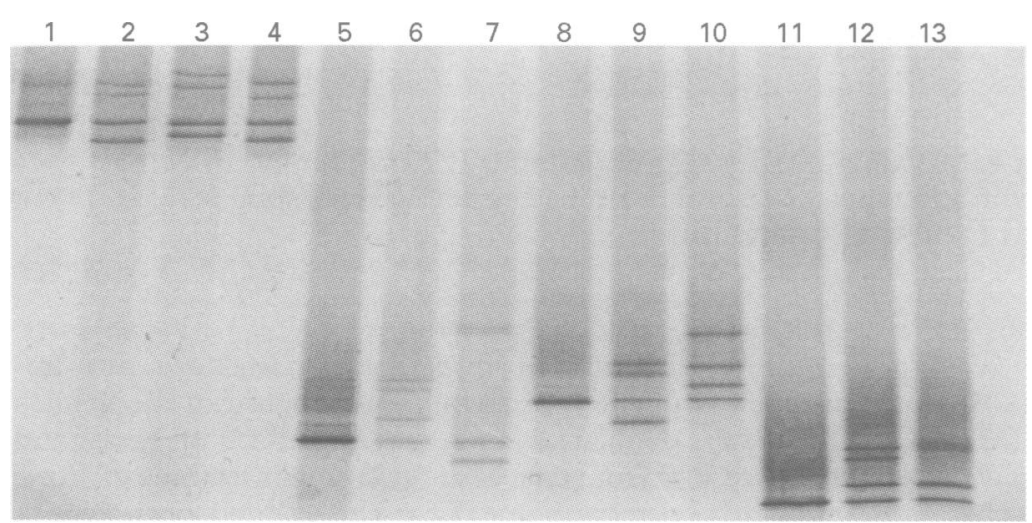

Figure 1 DGGE detection of c. 209T $\rightarrow$ C in the germline of a patient from a CS-like family. Control mutations from CS and BRR families are also included to display the sensitivity of this technique for the detection of PTEN mutations. Lane 1, wild type control (exon 3); lane 2, Y68H (exon 3); lane 3, IVS2-2A $\rightarrow G$ (exon 3); lane 4, c. $209 T \rightarrow C$ (exon 3); lane 5, wild type control (amplicon 5I, representing the 5' half of exon 5); lane 6, Q87X (amplicon 5I); lane 7, c.347-351delACAAT (amplicon 5I); lane 8, wild type control (amplicon 5II, representing the 3' half of exon 5); lane 9, C124R (amplicon 5II); lane 10, E157X (amplicon 5II); lane 11, wild type control (exon 7); lane 12, R233X (exon 7); lane 13, c.791ATins (exon 7).

the germline of families that did not meet the strict diagnostic criteria for CS determined by the International Cowden Consortium. ${ }^{2}{ }^{19}$ The phenotypes of these families were, minimally, breast and non-medullary thyroid cancers, and, maximally, a sum of phenotypes falling just short of the Consortium Criteria for CS.

\section{Material and methods}

PATIENTS

Members of 64 unrelated CS-like families were collected for analysis (table 1). These CS-like families were defined as families or people that have some, but not all, of the features of CS and do not meet the operational diagnostic criteria of the International Cowden Consortium. Minimally, these CS-like families contained at least one member with both non-medullary thyroid cancer and at least one other related member with breast cancer diagnosed at any age. They also could comprise subjects with both breast cancer and non-medullary thyroid cancer. Alternatively, families could be made up of either breast or non-medullary thyroid cancer and other features of CS, such as trichilemmomas, without meeting the consortium criteria for CS.

The diagnostic criteria for classical CS used in this study has been previously described by the Consortium. ${ }^{219}$ In brief, the diagnosis of CS requires that a patient or family meet a combination of pathognomonic major and minor criteria. Major criteria include breast cancer, non-medullary thyroid cancer (especially follicular thyroid carcinoma), macrocephaly ( $\geqslant 97$ th centile), and Lhermitte-Duclos disease (LDD), which is a dysplastic gangliocytoma of the cerebellum that can cause seizures, tremors, and poor coordination. Hamartomas of the skin, including trichilemmomas (benign tumours of the hair follicle infundibulum) and mucocutaneous papillomatous papules (for ex- $c$ ample, scrotal tongue), are diagnostic if there are six or more papules, with three or more being trichilemmomas. Minor criteria include benign $\frac{1}{D}$ thyroid lesions such as multinodular goitre and $\stackrel{\mathbb{P}}{\rightarrow}$ adenomas, fibrocystic breast disease, mental $\vec{\Rightarrow}$ retardation (IQ $\leqslant 75)$, gastrointestinal hamartomas, lipomas, fibromas, and genitourinary tumours or malformations. Individual people or $\frac{\bar{c}}{\bar{D}}$ families would be diagnosed with CS if they have $\frac{\vec{D}}{\vec{D}}$

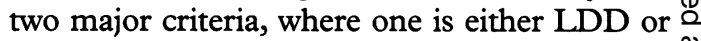
macrocephaly, one major with three minor के criteria, or four minor criteria. No patients in $\overrightarrow{0}$ this study fulfilled these criteria. Constitutional $\vec{\overrightarrow{ }}$ DNA was extracted from blood leucocytes using standard, previously described methods. ${ }^{34}$ Approval for the use of human subjects in this study was obtained under IRB approved protocol ô 94-138 (Dana-Farber Cancer Institute).

DENATURING GRADIENT GEL ELECTROPHORESIS (DGGE) AND TEMPORAL TEMPERATURE GEL ELECTROPHORESIS (TTGE)

A combination of DGGE and TTGE was performed for all nine exons of PTEN. GC $\stackrel{\Phi}{\rightrightarrows}$ clamped primer sequences, PCR conditions, $\underset{\mathbb{D}}{\mathbb{D}}$ and DGGE conditions have been previously described, ${ }^{35}$ with the exception of primers for $\&$ exons 2 and 4 . Exon 2 and 4 primer sequences, with GC clamps added, were as follows: exon 2, 2F, 5'-CGT CCC GCG TTT GAT TGC TGC ATA TTT CAG-3' and 2R, 5'-CGC के CCG CCG CGC CCC GCG CCC GTC CCG CCG CCC CCG CCC GTC TAA ATG AAA ACA CAA CAT G-3'; exon 4, 4F, 흠 5'-CGC CCG CCG CGC CCC GCG CCC GTC CCG CCG CCC CCG CCC GAA ATA ATA AAC ATT ATA AAG ATT CAG GCA ATG-3' and 4R, 5'-GAC AGT AAG ATA CAG TCT ATC-3'. Split exon 5 primers with GC clamps and conditions for mutation detection have been previously reported. ${ }^{26}$

TTGE is a mutation detection technique using the basic PCR fragment denaturation 을 principles of DGGE. The major difference between these methods is that a temperature gradient, rather than a chemical gradient of $N$ varying urea and glycerol percentages, is used for strand separation of the GC clamped homoand heteroduplexed PCR products by generating a linear temperature gradient over the length 2 of the electrophoresis run (Bio-Rad Laborato- $Q$ ries, Hercules, CA). One or $0.75 \mathrm{~mm}$ thick gels of $10 \%$ polyacrylamide:bis (37.5:1) (Bio-Rad Laboratories) and $7 \mathrm{~mol} / \mathrm{l}$ urea (Bio-Rad Laboratories) were run using the DCode ${ }^{\mathrm{TM}}$ Universal $\stackrel{\mathbb{D}}{\mathbb{D}}$ Mutation Detection System (Bio-Rad Labora- $\frac{\Omega}{\mathbb{D}}$ tories). Electrophoresis was performed at $130 \mathrm{~V} \Omega$ for six hours with a temperature gradient of $46-58^{\circ} \mathrm{C}$ and a ramp rate of $2^{\circ} \mathrm{C}$ per hour. $\delta$ TTGE fragments were visualised under ultraviolet transillumination after the gel was stained 흘 with ethidium bromide (Bio-Rad Laboratories).

Both DGGE and TTGE have proven high accuracy in detecting mutations in general and specifically in detecting known PTEN mutations from CS patients (fig 1). 
SEQUENCE ANALYSIS

Exons which showed DGGE and TTGE variants underwent direct sequence analysis. The PCR primers and reaction conditions have been described elsewhere. ${ }^{7142835}$ PCR products were gel isolated and purified using the Wizard PCR Preps DNA Purification System (Promega, Madison, WI). Direct sequencing of these products was performed using the ABI Prism dye terminator cycle sequencing ready reaction kit (Perkin-Elmer Corp. Norwalk, CT). Cycle sequencing products were electrophoresed on $6 \%$ Long ranger gels (FMC Bioproducts, Rockland, $\mathrm{ME}$ ) and analysed on an Applied Biosystems model 373A automated DNA sequencer (Perkin-Elmer Corp).

PTEN POLYMORPHISM ANALYSIS

A previously identified intronic polymorphic site in PTEN, IVS8+32G/T, was analysed in a single affected member from each CS-like family to investigate hemizygosity at the PTEN locus in mutation negative families. This site is moderately heterozygous, with an earlier report finding $50 \%$ of samples to be informative. ${ }^{28}$ Potential hemizygosity was assessed by the amplification of exon 8 and flanking intronic sequence and digestion with the restriction endonuclease HincII under conditions suggested by the manufacturer (New England Biolabs, Beverly, MA).

\section{Results}

PTEN MUTATION ANALYSIS

A missense point mutation, c.209T $\rightarrow C$ (L70P), predicted to affect splicing was identified in a single affected patient ( 1 of $64,2 \%$ ) (fig 1). This mutation was not identified in 100 normal alleles. When this occult germline PTEN mutation was identified, the family history was reassessed (fig 2). The subject analysed for this study, III.1, developed follicular thyroid carcinoma at the age of 31 . His

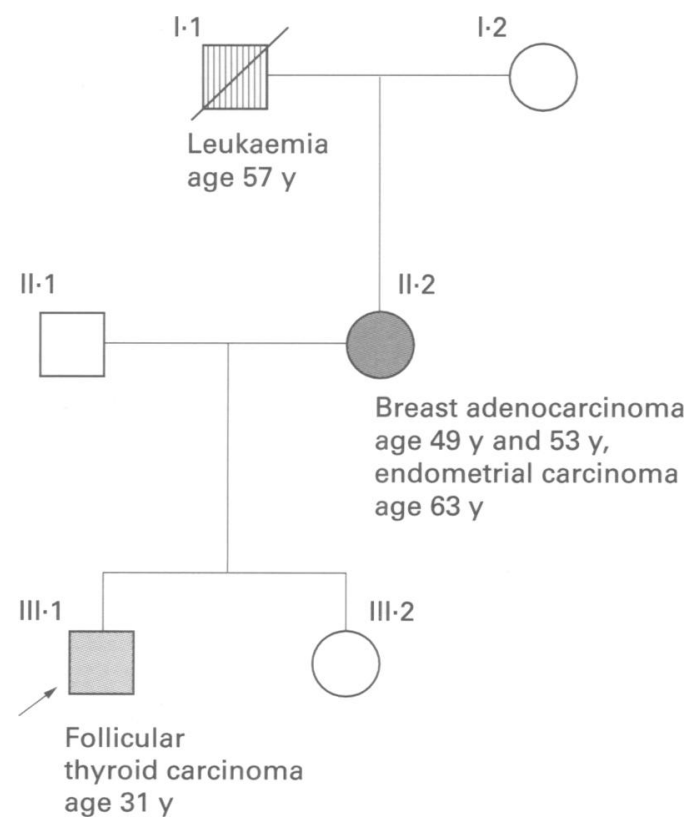

Figure 2 Pedigree of CS-like family with the occult germline PTEN mutation. c. 209T $\rightarrow C$ was identified in DNA extracted from blood leucocytes from patient III. 1 who presented with follicular thyroid carcinoma. mother, II.2, had breast adenocarcinoma diagnosed at the age of 49 and again at 53. She also had endometrial carcinoma diagnosed at 63 years. Careful clinical assessment of these two subjects was unable to identify macrocephaly, skin lesions typical of CS, or scrotal tongue. The maternal grandfather, I.1, was diagnosed with leukaemia at the age of 57 . Unfortunately, family members other than III. 1 were unavailable for analysis. Fresh tumour from III.1, which would have allowed us to study the putative aberrant splicing effect of this mutation, was also unavailable. No mutations were identified in the other 63 unrelated CS-like families.

\section{PTEN POLYMORPHISM ANALYSIS}

Forty-eight percent (30 of 63) of unrelated subjects from PTEN mutation negative CSlike families were found to be heterozygous at the IVS $8+32 \mathrm{~T} / \mathrm{G}$ site. This analysis would suggest that, at least in these families, gross germline deletion of PTEN can be excluded.

\section{Discussion}

An occult germline PTEN mutation, c. $209 \mathrm{~T} \rightarrow \mathrm{C}$ at the last nucleotide of exon 3 was found in one of $64(2 \%)$ CS-like families. This family's cancers, comprising leukaemia, which may or may not be related, adenocarcinoma of the breast, endometrial carcinoma, and follicular thyroid carcinoma, together do not meet the International Cowden Consortium Criteria used for the diagnosis of CS in this study. However, we cannot exclude the possibility that this family represents a case of low penetrance CS. The family with PTEN mutation in this study contrasts with that in a recent study that reported a PTEN mutation in a family initially classified as having breast and thyroid tumours only but reclassified as CS after mutation analysis led to closer clinical assessment. ${ }^{36}$ Closer clinical assessment of the family presented in the current study did not identify additional features of CS.

In the remaining families where no occult germline mutations were identified, it is highly unlikely that these mutations would have gone undetected. Both DGGE and TTGE are highly sensitive mutation detection techniques ${ }^{37}$ and both have been shown consistently to detect known PTEN mutations and other sequence polymorphisms (Marsh and Eng, unpublished data, 1998; fig 1). Further, because at least one affected member from nearly half of these mutation negative families was heterozygous at the IVS $8+32 \mathrm{~T} / \mathrm{G}$ polymorphism, whole gene deletion is unlikely, at least in these families.

In CS, while missense and truncating mutations are scattered largely along the entirety of PTEN, a mutational "hot spot" exists in exon 5, which contains the PTPase core motif at codons 122-132. ${ }^{76-18}$ Thus, many mutations in CS are predicted to disrupt the phosphatase function of this protein. Interestingly, the mutation identified in exon 3 falls in the $\mathrm{N}$-terminal half of the PTEN protein that has been shown to have some sequence similarities to the cytoskeletal proteins tensin and auxilin. 
Specifically, the leucine residue at codon 70 that is altered by this $\mathrm{T}$ to $\mathrm{C}$ point mutation (L70P) is conserved in both bovine auxilin and chicken tensin. ${ }^{14}$ Thus, it is possible that this mutation may be affecting the phosphatase function of this protein, as one may predict if this putative splice site mutation leads to a truncated protein, and may also function to disrupt normal cellular motility and cell-cell interactions.

Whether germline PTEN mutations are associated with CS and related inherited hamartoma syndromes (Bannayan-RuvalcabaRiley syndrome, (BRR, MIM 153480) and juvenile polyposis syndrome (JPS, MIM 174900)), as well as syndromes comprising partial CS phenotypes, is largely unknown. Before the identification of PTEN as the CS gene, it was not inconceivable that the three related hamartoma syndromes and CS-like syndromes were all associated with different mutations in a single gene. We have shown that germline PTEN mutations are associated with the great majority, approximately $80 \%$, of classical CS families. ${ }^{78}$ Nelen et al ${ }^{17}$ identified PTEN mutations in $47 \%$ of CS cases studied. One other study of $23 \mathrm{CS}$ families identified only $13 \%$ of families with germline PTEN mutation. ${ }^{16}$ This was perhaps not surprising as limited linkage information in these families suggested the possibility of genetic heterogeneity in CS, even though initial studies of a group of $12 \mathrm{CS}$ families showed no evidence for heterogeneity. ${ }^{19}$

We have also shown that germline PTEN mutations account for at least a proportion of $B R R$, which is characterised by macrocephaly, lipomatosis, thyroid dysfunction, hamartomatous polyps of the gastrointestinal tract, and pigmented macules of the glans penis, but without a known predisposition to breast and thyroid cancer. ${ }^{18}{ }^{38}$ How mutations in a single gene, at times identical, ${ }^{18}{ }^{38}$ can function to predispose to two overlapping but apparently distinct syndromes, one with malignancy and one without, remains to be elucidated.

Disparate reports concerning the third hamartoma syndrome, JPS, and PTEN mutation or deletion have recently been published..$^{35}{ }^{36}{ }^{39-41}$ A putative JPS locus, JP1, at 10q22-24 was initially thought to encompass PTEN, although fine structure mapping placed this locus slightly centromeric of PTEN. ${ }^{42}$ Subsequently, the 10q22-24 region was excluded as a putative JPS locus by linkage analysis in eight JPS families. ${ }^{35}$ Screening of PTEN in 21 classical JPS families and 16 cases of sporadic JPS did not identify any germline mutations. ${ }^{35}{ }^{39}$ In contrast, PTEN mutation has been reported in four patients with "juvenile polyposis", ${ }^{361}$ although the clinical diagnosis of classic juvenile polyposis in these cases is questionable. Given these genetic data and the phenotypic overlap of these syndromes, we can say with some confidence that if a germline PTEN mutation were detected in a person previously thought to have "juvenile polyposis", then the diagnosis needs to be revised, as that person is likely to have either CS or BRR.
Along the same lines, we have now investigated a cohort of families, each of which contains some of the component tumours of $c$ CS but do not meet the Consortium diagnostic $\frac{\bar{d}}{\mathbb{D}}$ criteria for CS. Only one such family was found to have an occult germline PTEN mutation, $\Phi$ arguing that such germline alterations play a $\Phi$ minor role in families that do not meet the strict CS diagnostic criteria. Nonetheless, this $\stackrel{\vec{S}}{\stackrel{9}{ }}$ finding is significant for three reasons. Firstly, it suggests that the operational diagnostic criteria $\frac{\bar{F}}{\bar{D}}$ for CS established by the International Cow- $\frac{N}{\vec{D}}$

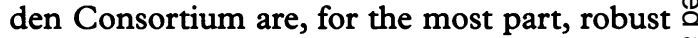
and are useful for identifying PTEN mutation क positive CS families. Secondly, we must also $\overrightarrow{0}$ conclude from our data that other genes are involved which lend susceptibility to a CS-like $\vec{\omega}$ disease and to site specific breast and non- $\frac{\widehat{T}}{3}$ medullary thyroid cancer. Thirdly, for non-CS subjects identified with occult PTEN muta- $w$ tions, albeit uncommonly, there are important implications for future hamartoma/cancer development that should impact on surveillance. $\stackrel{\infty}{\rightarrow}$

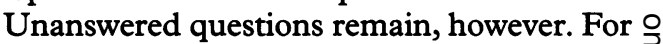
example, are CS-like families without germline PTEN mutations at any less risk of cancer than $z$ those with mutations? Preliminary genotypephenotype analyses suggest that classical CS families without germline PTEN mutations are $\Phi$ at lower risk of developing malignant breast $\vec{\theta}$

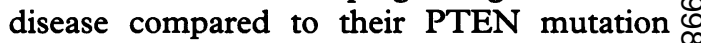
positive counterparts. ${ }^{18}$ By extrapolation, it would seem that PTEN mutation negative CS-like families should be at decreased risk of developing breast cancer. Unfortunately, this study was unable to confirm this clinically relevant extrapolation. We can conclude, however, that in the majority of cases, germline PTEN mutations lead specifically to a CS or BRR phenotype and that the phenotype of CS-like families is, for the most part, caused by unknown mechanisms.

We would like to thank the patients and families who participated in this study, and Dr Oliver Gimm for critical read- $O$ ing of this manuscript. Ms Elaine Krekonis is acknowledged for assistance with the patients. The Molecular Biology Core Facility at the Dana-Farber Cancer Institute, Boston is acknowl- $O$ cd edged for running sequencing gels. This study was supported by $I$ (to PLMD and CE), the American Cancer Society (RPG 97-064-02VM), the Barr Investigatorship, and a Breast Cancer Research Grant (34088PP1009) from the Massachusetts Department of Public $O$ Health (to CE). CE is the Lawrence and Susan Marx Investigator in Human Cancer Genetics.

1 Starink TM, van der Veen JPW, Arwert F, et al. The Cowden syndrome: a clinical and genetic study in 21 patients. Clin Genet 1986;29:222-33.

2 Eng C. Cowden syndrome. $\mathcal{F}$ Genet Counsel 1997;6:9181-92.

3 Malkin D, Li FP, Fraumeni JF Jr, et al. Germ-line p53. mutations in a familial syndrome of breast cancer, sarcomas, and other neoplasms. Science 1990;250:1233-8.

Srivastava S, Zou Z, Pirollo K, Blattner W, Chang EH. Germ-line transmission of a mutated 053 gene in a cancerGerm-line transmission of a mutated pS3 gene in a cancerprone family with Li-Fraumeni syndrome. Nature 1990; $\mathbb{D}$

5 Miki Y, Swensen J, Shattuck-Eidens D, et al. A strong candidate for the breast and ovarian cancer susceptibility gene $B R C A 1$. Science 1994;266:66-71.

6 Wooster R, Bignell G, Lancaster J, et al. Identification of the $\frac{8}{6}$ breast cancer susceptibility gene BRCA2. Nature 1995;378: 789-92.

7 Liaw D, Marsh DJ, Li J, et al. Germline mutations of the PTEN gene in Cowden disease, an inherited breast and thyroid cancer syndrome. Nat Genet 1997;16:64-7.

8 Easton DF, Bishop DT, Ford D, Crockford GP, Consortium BCL. Genetic linkage analysis in familial breast and BCL. Genetic linkage analysis in familial breast and
ovarian cancer: results from 214 families. The Breast Canovarian cancer: results from 214 families. The Breast Cancer

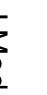


9 Wooster R, Neuhausen SL, Manjion J, et al. Localization of a breast cancer susceptibility gene, $B R C A 2$, to chromosome 13q12-12. Science 1994;265:2088-90.

10 Eng C. From bench to bedside...but when? Genome Res 1997;7:669-72.

11 Varley JM, McGown G, Thorncroft M, et al. Germ-line mutations of TP53 in Li-Fraumeni families: an extended study of 39 families. Cancer Res 1997;57:3245-52.

$12 \mathrm{Li} \mathrm{J}$, Yen C, Liaw D, et al. PTEN, a putative protein tyrosine phosphatase gene mutated in human brain, breast, and prostate cancer. Science 1997;275:1943-7.

$13 \mathrm{Li} \mathrm{DM}$, Sun H. TEP1, encoded by a candidate tumor suppressor locus, is a novel protein tyrosine phosphatase regulated by transforming growth factor $\beta$. Cancer Res 1997;57 2124-9.

14 Steck PA, Pershouse MA, Jasser SA, et al. Identification of a candidate tumor suppressor gene, $M M A C 1$, at chromosome $10 \mathrm{q} 23.3$ that is mutated in multiple advanced cancers. Nat Genet 1997;15:356-62.

15 Myers MP, Stolarov JP, Eng C, et al. P-TEN, the tumor suppressor from human chromosome $10 \mathrm{q} 23$, is a dualspecificity phosphatase. Proc Natl Acad Sci USA 1997;94 9052-7.

16 Tsou HC, Teng DHF, Li Ping X, et al. The role of $M M A C 1$ mutations in early-onset breast cancer: causative in association with Cowden syndrome and excluded in $B R C A 1$ negative cases. Am $₹$ Hum Genet 1997;61:1036-43.

17 Nelen MR, van Staveren WCG, Peeters EAJ, et al. Germline mutations in the PTEN/MMAC1 gene in patients with mutations in the PTEN/MMAC1 gene in patient

18 Marsh DJ, Coulon V, Lunetta KL, et al. Mutation spectrum and genotype-phenotype analyses in Cowden disease and Bannayan-Zonana syndrome, 2 hamartoma syndromes with germline PTEN mutation. Hum Mol Genet 1998;7: 507-15.

19 Nelen MR, Padberg GW, Peeters EAJ, et al. Localization of the gene for Cowden disease to chromosome 10q22-23. Nat Genet 1996;13:114-16.

20 Liu W, James CD, Frederick L, Alderete BE, Jenkins RB. $P T E N / M M A C 1$ mutations and EGFR amplification in glioblastomas Cancer Res 1997;57:5254-7.

21 Teng DHF, Hu R, Lin $\mathrm{H}$, et al. MMAC1/PTEN mutations in primary tumor specimens and tumor cell lines. Cancer Res 1997;57:5221-5.

22 Rhei E, Kang L, Bogomolniy F, Federici MG, Borgen PI, Boyd J. Mutation analysis of the putative tumor suppressor gene PTEN/MMAC1 in primary breast carcinomas. Cancer Res 1997;57:3657-9.

23 Wang SI, Puc J, Li J, et al. Somatic mutations of PTEN in glioblastoma multiforme. Cancer Res 1997;57:4183-6.

24 Ahmed Rasheed BK, Stenzel TT, McLendon RE, et al. PTEN gene mutations are seen in high-grade but not in low-grade gliomas. Cancer Res 1997;57:4187-90.

25 Cairns P, Ookami K, Halachmi S, et al. Frequent inactivation of $P T E N / M M A C 1$ in primary prostate cancer. Cancer Res 1997;57:4997-5000.

26 Guldberg P, Straten PT, Birck A, Ahrenkiel V, Kirkin AF Zeuthen J. Disruption of the MMAC1/PTEN gene by dele- tion or mutation is a frequent event in malignant melanoma. Cancer Res 1997;57:3660-3.

27 Tashiro H, Blazes MS, Wu R, et al. Mutations in PTEN are frequent in endometrial carcinoma but rare in other common gynecological malignancies. Cancer Res 1997;57: 3935-40.

28 Dahia PLM, Marsh DJ, Zheng Z, et al. Somatic deletions and mutations in the Cowden disease gene, PTEN, in sporadic thyroid tumors. Cancer Res 1997;57:4710-13.

29 Risinger J, Hayes AK, Berchuk A, Barrett JC. PTEN/ $M M A C 1$ mutations in endometrial cancers. Cancer Res 1997;57:4736-8.

30 Funari FB, Lin H, Su Huang HJ, Cacenee WK. Growth suppression of glioma cells by PTEN requires a functional phosphatase catalytic domain. Proc Natl Acad Sci USA 1997;94:12479-84

31 Sakurada A, Suzuki A, Sato $M$, et al. Infrequent genetic alterations of the PTEN/MMAC1 gene in Japanese patients with primary cancers of the breast, lung, pancreas, kidney and ovary. Fpn $\mathcal{F}$ Cancer Res 1997;88:1025-8.

32 Ueda $\mathrm{K}$, Nishijima $M$, Inui $\mathrm{H}$, et al. Infrequent mutations in the PTENIMMAC1 gene among primary breast cancers. Fpn f Cancer Res 1997;89:17-21.

33 Chiariello E, Roz L, Albarosa R, Magnani I, Finocchiaro J. $P T E N / M M A C 1$ mutations in primary glioblastomas and short-term cultures of malignant gliomas. Oncogene 1998; 16:541-5.

34 Mathew CG, Smith BA, Thorpe K, et al. Deletion of genes on chromosome 1 in endocrine neoplasia. Nature 1987; 328:524-6.

35 Marsh DJ, Roth S, Lunetta KL, et al. Exclusion of PTEN and $10 \mathrm{q} 22-24$ as the susceptibility locus for juvenile and $10 \mathrm{q} 22-24$ as the susceptibility locus for

36 Lynch ED, Ostermeyer EA, Lee MK, et al. Inherited mutations in PTEN that are associated with breast cancer, Cowden disease, and juvenile polyposis. Am F Hum Genet 1997; 61:1254-60.

37 Eng C, Vijg J. Genetic testing: the problems and the promise. Nat Biotechnol 1997;15:422-6.

38 Marsh DJ, Dahia PLM, Zheng Z, et al. Germline mutations in PTEN are present in Bannayan-Zonana syndrome. Nat Genet 1997;16:333-4.

39 Riggins GJ, Hamilton SR, Kinzler KW, Vogelstein B. Normal PTEN gene in juvenile polyposis. $\mathcal{F}$ Neg Obstet Genet Oncol 1998;1:1.

40 Tsuchiya KD, Wiesner G, Cassidy SB, Limwongse C, Boyle JT, Schwartz S. Deletion 10q23.2-q23.33 in a patient with gastrointestinal juvenile polyposis and other features of a gastrointestinal juvenile polyposis and other features of a 18.

41 Olschwang S, Serova-Sinilnikova OM, Lenoir GM, Thomas G. PTEN germline mutations in juvenile polyposis coli. Nat Genet 1998;18:12-14.

42 Jacoby RF, Schlack S, Cole CE, Skarbek M, Harris C, Meisner LF. A juvenile polyposis tumor suppressor locus at $10 \mathrm{q} 22$ is deleted from nonepithelial cells in the lamina propria. Gastroenterology 1997;112:1398-403. 